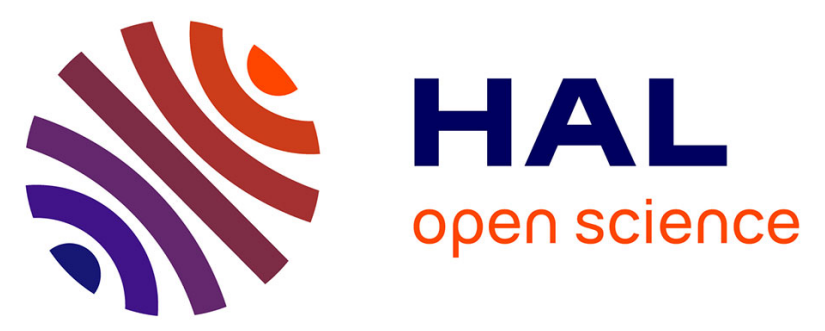

\title{
Reduced Regional Cortical Thickness Rate of Change in Donepezil-Treated Subjects With Suspected Prodromal Alzheimer's Disease
}

Enrica Cavedo, Bruno Dubois, Olivier Colliot, Simone Lista, Bernard Croisile, Guy Louis Tisserand, Jacques Touchon, Alain Bonafe, Pierre J. Ousset, Olivier Rouaud, et al.

\section{To cite this version:}

Enrica Cavedo, Bruno Dubois, Olivier Colliot, Simone Lista, Bernard Croisile, et al.. Reduced Regional Cortical Thickness Rate of Change in Donepezil-Treated Subjects With Suspected Prodromal Alzheimer's Disease. Journal of Clinical Psychiatry, 2016, 10.4088/JCP.15m10413 . hal-01388951

\section{HAL Id: hal-01388951 \\ https://inria.hal.science/hal-01388951}

Submitted on 12 Nov 2016

HAL is a multi-disciplinary open access archive for the deposit and dissemination of scientific research documents, whether they are published or not. The documents may come from teaching and research institutions in France or abroad, or from public or private research centers.
L'archive ouverte pluridisciplinaire HAL, est destinée au dépôt et à la diffusion de documents scientifiques de niveau recherche, publiés ou non, émanant des établissements d'enseignement et de recherche français ou étrangers, des laboratoires publics ou privés. 


\section{Reduced regional cortical thickness rate of change in donepezil treated subjects with suspected prodromal Alzheimer's disease}

Enrica Cavedo a,b,c,d,e*, PhD, Bruno Dubois a,b,c, MD, Olivier Colliot f,g,h,i,j, PhD, Simone Lista ${ }^{a, b, c, k}$, PhD, Bernard Croisile ', MD, Guy Louis Tisserand m, MD, Jaques Touchon ${ }^{n}$, MD, Alain Bonafe ${ }^{n}, M^{2}$, Pierre J Ousset ${ }^{\circ}$, MD, Olivier Rouaud P, MD, Fréderic Ricolfi ${ }^{p}, M D$, Alain Vighetto ${ }^{q}, M D$, Florence Pasquier ${ }^{r}, M D$, Samantha Galluzzi e, MD, Christine Delmaire s, MD, Mathieu Ceccaldi t, MD, Nadine Girard t, MD, Stéphane Lehericy b,c, u, MD, Françoise Duveau v, MD, Marie Chupin f,g,h,i,j, PhD, Marie Sarazin w, MD, Didier Dormont ${ }^{\mathrm{f}, \mathrm{g}, \mathrm{h}, \mathrm{i},-\mathrm{x}}$, MD, and Harald Hampel a,b,c,k* $\mathrm{k}^{\star}, \mathrm{MD}, \mathrm{PhD}$ for the "Hippocampus Study Group"

a Institut de la Memoire et de la Maladie d'Alzheimer (IM2A), Departement de Neurologie, Hôpital de la Pitie-Salpêtriere, AP-HP, Paris, France

b INSERM U1127, Institut du Cerveau et de la Moelle Épinière (ICM), Paris, France

c Sorbonne Universites, Universite Pierre et Marie Curie-Paris 6, Paris, France

d CATI multicenter neuroimaging platform (cati-neuroimaging.com), France;

e IRCCS Istituto Centro San Giovanni di Dio-Fatebenefratelli, Brescia, Italy

${ }^{f}$ Inserm, U1127, F-75013, Paris, France

g CNRS, UMR 7225 ICM, 75013, Paris, France

h Sorbonne Universités, UPMC Univ Paris 06, UMR S 1127, F-75013, Paris, France i Institut du Cerveau et de la Moelle épinière, ICM, F-75013, Paris, France j Inria, Aramis project-team, Centre de Recherche Paris-Rocquencourt, France ${ }^{k}$ AXA Research Fund \& UPMC Chair, Paris, France

' Department of Neuropsychology, Hôpital Neurologique Pierre Wertheimer, Lyon, France

m Hôpital Neurologique Pierre Wertheimer, Lyon, France

${ }^{n} \mathrm{CHRU}$, Gui de Chauliac, Montpellier, France

- Centre Mémoire / Centre de Recherche Clinique - Gérontopôle, Hôpital Casselardit, Toulouse, France

${ }^{p}$ Hôpital General, Dijon, France 
q Lyon 1 university, Hospices Civils de Lyon, hôpital neurologique, service de neurologie D, Lyon, France

rUniversité de Lille, Inserm U1171, CHU, centre mémoire 59000 Lille France

${ }^{s}$ Université de Lille, Inserm U1171, Service de Neuroradiologie, Hôpital Roger

Salengro, Lille, France

${ }^{\mathrm{t}}$ Hôpital de la Timone, Marseille, France

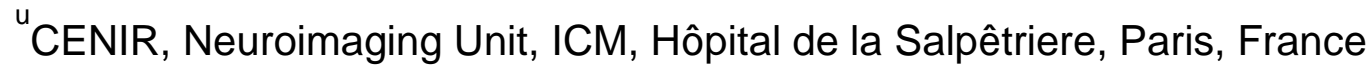

${ }^{\vee}$ Eisai SAS, La Defense 2 Cedex, France

w Neurologie de la Mémoire et du Langage, Université Paris Descartes, Sorbonne

Paris Cité, INSERM UMR S894, Centre Hospitalier Sainte Anne, Paris, France

${ }^{x}$ Neuroradiology Department, Hôpital de la Salpêtriere, Paris, France

\section{${ }^{*}$ Corresponding authors:}

Enrica Cavedo and Harald Hampel,

Université Pierre et Marie Curie, Paris 06,

Département de Neurologie,

Institut de la Mémoire et de la Maladie d'Alzheimer (IM2A),

Hôpital de la Pitié-Salpêtrière,

47 Boulevard de l'Hôpital, 75013 Paris, France.

Tel.: +33 14261925

Fax: +33142167516

E-Mails: enrica.cavedo@gmail.com; harald.hampel@icm-institute.org;

harald.hampel@med.uni-muenchen.de 


\section{Abstract}

OBJECTIVES: Cortical thinning, previously identified during prodromal stages of AD, is a "candidate" biomarker implemented in $A D$ clinical therapy trials. We have investigated the effect of donepezil treatment on cortical thickness in mild cognitively impaired subjects with the amnestic syndrome of the hippocampal type, a prodromal at risk group for progression to $A D$ dementia.

METHODS: Longitudinal analysis of a community-based multi-centric suspected prodromal AD cohort (92 placebo versus 81 donepezil) enrolled in a double-blind, randomized, placebo-controlled parallel group design using donepezil (10mg/day) has been conducted. The study started in November 2006 and was concluded in August 2010. All subjects underwent two brain structural Magnetic Resonance Imaging (MRI) scans, at baseline and at the end of the trial. Structural MRI images have been processed using the automated pipeline for longitudinal segmentation and surface reconstruction implemented in FreeSurfer. The primary outcome measure of this posthoc study was the cortical thickness annualized percentage change (APC).

RESULTS: The donepezil group exhibited reduced APC cortical thinning compared to placebo in the Rostral Anterior Cingulate (right: $p=0.048$; and left: $p=0.032$ ), the Orbitofrontal (right: $p=0.012$ and left $p<0.048)$, and the right Inferior Frontal $(p=$ $0.022)$ cortices and in the right Insula $(p=0.010)$. These results were not statistically significant after Bonferroni correction likely due to insufficient power for cortical thickness measurements in the study group powered for the predefined hippocampus outcome.

CONCLUSIONS: Our findings support the hypothesis that cortical thickness is a reliable candidate surrogate outcome in early pre-dementia AD trials. In addition, donepezil treatment may have an impact on cortical structure/morphology in areas 
innervated by the medial and lateral cholinergic pathways.

Clinical Trial Registration: ClinicalTrials.gov NCT00403520

Key words: Cortical Thickness, Alzheimer's Disease, MRI, Donepezil, Cholinergic system, Clinical Trials, prodromal AD, Mild Cognitive Impairment, Progression 


\section{Introduction}

The current approved treatments with cholinesterase inhibitors are generally considered as moderately and only symptomatically beneficial for late-stage $A D$ dementia patients ${ }^{1}$. Evidence of potential biological and disease-modifying properties of cholinesterase inhibitors is controversially and critically discussed. No agent tested in randomized clinical trials in Mild Cognitive Impairment $(\mathrm{MCl})$ has met its primary efficacy objectives, measured by clinical rating instruments ${ }^{2-10}$.

To date, only one study investigating the use of in vivo biomarkers of $A D$ in Donepezil clinical trial has been conducted ${ }^{11}$. On the other hand, further clinical studies on structural imaging markers in $\mathrm{MCl}$ subjects have reported conflicting results ${ }^{12-14}$.

Although cortical-thickness represents a promising structural imaging end point in clinical trials, as it offers a direct assessment of effect sizes expressed in a meaningful metric, ${ }^{15-17}$ no study has investigated the donepezil effect on the cortical mantle yet.

This is a post hoc study based on the data collected in the context of the Hippocampus Study Clinical Trial (NCT00403520). The first aim of the trial was to investigate the effect of Donepezil treatment using the hippocampal rate of changes as primary outcome ${ }^{14}$. Here, we are going to consider the impact of donepezil treatment on the thickness rate of change in suspected prodromal $A D$ patients diagnosed using the Free and Cued Selective Reminding Test (FCSRT), a test allowing prognostication of prodromal $\mathrm{AD}$ within the group of $\mathrm{MCl}$ individuals ${ }^{18,19}$. 


\section{Methods}

Study Population

Participants included in the present study were all the patients who performed a baseline and a follow-up MRI scan during the Hippocampus Study Clinical Trial (NCT00403520) ${ }^{14}$. The primary efficacy outcome of the Hippocampus Study was the annualized percentage change (APC) of total hippocampal volume measured by an automated segmentation method. In this post hoc analysis, we explored the effect of Donepezil on the cortical thickness. Each patients received at least 1 dose of doubleblind study medication, and had baseline and follow-up clinical and Magnetic Resonance Imaging (MRI) assessment.

The details of the patient characteristics have been previously described ${ }^{14}$. Briefly, a total of 332 patients were screened within the national network of Memory Resources and Research Centres (MRRC) consisting of 28 regional university expert centres with neurologists, geriatricians, neuropsychologists, biological and neuroimaging resources in each centre.

Inclusion criteria were: 1) more than 50 years of age; 2) a progressive hippocampal amnestic syndrome defined by Free Recall $\leq 17$ or Total Recall $<40$ on the FCSRT; and 3) no dementia with a CDR stage of 0.5 and preserved cognition and functional performance. Subjects who met the eligibility criteria were enrolled in the randomization phase beginning with Visit 1. From the total population of individuals randomized in the clinical trial (103 Placebo and 113 Donepezil), for the present study we considered exclusively patients who performed MRI at baseline and at the end of the treatment (Placebo=92 and Donepezil= 82).

\section{Study Design}


This was a multi-centre double-blind, randomized, placebo-controlled trial with a treatment period of 12 months. The sample power of the study was calculated according to the primary aim of the Hippocampus Study Clinical Trial, and not for the aim of the present study. The study started in November 2006 and was concluded in August 2010. At baseline visit, patients underwent a baseline MRI scan and a cognitive evaluation including: the Alzheimer's Disease Assessment Scale-cognitive subscale, $\mathrm{MCl}$ version (ADAS-COG-MCI) ${ }^{20}$, Mini-Mental State Examination (MMSE), Modified Isaacs test score, California Verbal Learning Test (CVLT) ${ }^{21}$, Trial Making Tests (TMT) $A$ and $\mathrm{B}^{22}$, and the Benton Test ${ }^{23}$. Following baseline evaluation, patients were randomly assigned to one group out of two, corresponding to either active treatment or placebo ( 1 capsule of 5 -mg donepezil daily for Weeks 0 to 6 , then 2 capsules of 5-mg donepezil [i.e., $10 \mathrm{mg}$ ] daily from Week 6 to Month 12 for doubleblind treatment; or 1 placebo capsule daily for Weeks 0 to 6 , then 2 capsules daily from Week 6 to Month 12 for double-blind treatment, respectively). The study protocol was approved by the institutional review board of each site, and informed consent was obtained from all subjects.

\section{Acquisition of MR images}

Brain MRI scans were acquired in each centre at baseline and after at the end of treatment period. All the MRI were performed using 1.5 Tesla or 3 Tesla MRI scanners qualified by the central MRI analysis core at the Cogimage team, Centre de Recherche de l'institut du Cerveau et de la Moelle Épinière (CRICM). The sequences used in the present manuscript were 3D T1-weighted images. The 3D T1-weighted scan parameters for scans at $1.5 \mathrm{~T}$ were: $\mathrm{TR}=10 \mathrm{~ms}, \mathrm{TE}=$ minimum, flip angle: 10, PREP time: 600 , bandpass: $12.50 \mathrm{khz}$, axial orientation; $1.3 \mathrm{~mm}$ slice thickness, contiguous (124 slices), Imaging matrix size: $256 \times 256$, Field of view: $240 \times 240$; for scans at 3T 
were: $\mathrm{TR}=3.9 \mathrm{~ms}, \mathrm{TE}=2100 \mathrm{~ms}$, flip angle: 15 , PREP time: $1100 \mathrm{~ms}$, sagittal orientation with $1 \mathrm{~mm}$ slice thickness, contiguous (144 slices), Acquisition matrix size: $256 \times 192$, Field of view: $256 \times 192$. The expert neuroradiologist in charge of quality control verified artifacts of movements, ringing, wrap around and metal artifacts, moreover they attested that the sequences were acquired according to protocol parameters. After the quality control of images, one 3D T1-weighted scan was identified as corrupted and it was excluded from the all statistical and the neuroimaging analysis.

Cortical Thickness Reconstruction and Surface Analysis

To extract reliable thickness estimates, images were automatically processed with the longitudinal stream ${ }^{24}$ in FreeSurfer (http://surfer.nmr.mgh.harvard.edu/) version 5.1. Details on the longitudinal pipeline used to analyzing data are described at the following link (https://surfer.nmr.mgh.harvard.edu/fswiki/LongitudinalProcessing). Specifically an unbiased within-subject template space and image is created using robust, inverse consistent registration ${ }^{25}$. Several processing steps, such as skull stripping, Talairach transforms, atlas registration as well as spherical surface maps and parcellations are then initialized with common information from the within-subject template, significantly increasing reliability and statistical power ${ }^{26}$. Based on gyral and sulcal anatomy, the cortex was segmented using the Desikan-Killiany Atlas ${ }^{27}$. For each of cortical regions, mean cortical thickness was calculated as the distance (in $\mathrm{mm}$ ) between the pial and gray/white matter surfaces. A quality control of data was performed, the Pial and the white matter Surfaces were checked in order to remove any non brain tissue and non-white matter tissue respectively. The cortical thickness was smoothed with $10 \mathrm{~mm}$ FWHM Gaussian kernel to improve the signal- 
to-noise ratio and statistical power.

\section{Statistical Analysis}

Demographic, clinical and neuropsychological features were compared between subject groups (Placebo vs Donepezil). Chi-square test was performed on categorical variables, while the one-way analysis of variance (ANOVA) was utilized for continuous variables. Since the assumption of normality was violated, baseline cortical thicknesses measures were transformed according to a Two-Step Approach for Transforming Continuous Variables to Normal. The first step involves transforming the original variable toward statistical uniformity (i.e., satisfies the preponderance of diagnostics tests for uniformity) by calculating the fractional rank of each score, then fractional rank obtained from the previous step were transformed to normal using the inverse normal distribution function ${ }^{28}$. Normalized baseline cortical thickness measures have been compared between groups using the ANOVA. Annualized Percentage Change (APC) of cortical thickness, in all the regions extracted from FreeSurfer pipeline, were computed as follows:

$$
A P C=\frac{\text { change from baseline }}{\text { value at baseline }} \times \frac{365}{\text { MRI delay }} \times 100
$$

Then, the cortical thickness APC were compared between placebo and donepezil using the Wilcoxon-Mann-Whitney test.

The cortical thickness APC comparisons were supplemented post hoc by the linear mixed effects regression model with random intercepts, controlling for scanner platforms, to compare the difference in the cortical thickness change between placebo and donepezil patients in relation to treatment. Bonferroni correction for 
multiple comparisons was applied to the cortical thickness APC comparisons and to the mixed effect regression model results. The statistical analyses were performed using SPSS v.22.00.

Surface analyses were performed using MATLAB (http://fr.mathworks.com) and the QDEC toolbox of FreeSurfer (www.surfer.nmr.mgh.harvard.edu). One-year changes in bilateral cortical structures were investigated using the Linear Mixed Effects Models. The results were projected onto the template. The surface analyses were rethresholded using a two-stage false discovery rate of 0.05 (FDR2 $<0.05$ ).

\section{Power analysis}

Since the original Hippocampus Study trial was not designed for examine cortical thickness, an estimation of samples size needed to detect a thickness change of a certain number of millimetres between placebo and treatment patients was estimated. Sample size requirements were estimated using the following formula:

$2 \times K \times\left(\frac{\sigma}{\mu 1-\mu 2}\right)^{2}$, where $K=$ constant, which is a function of $\alpha(0.05)$ and $\beta=0.2$ $(80 \%) ; \sigma$ is the standard deviation of cortical thickness APC in the untreated group, $\mu$ are the mean values of cortical thickness APC in the treatment and placebo groups. The following modification was included: the sample size thus derived was increased by $10 \%$ to allow for losses at follow-up. 


\section{Results}

From the total sample of 316 individuals (103 Placebo and 113 Donepzil) randomized in the trial, 174 individuals (92 Placebo and 82 Donepezil) underwent both baseline and follow-up MRI scans. One subject of them was excluded from analysis due to a corrupted scan.

No significant differences were found at baseline sociodemographic as well as in cognitive features between groups (Table 1). The Apolipoprotein $\mathrm{E}(A P O E)$ genotype was present in $18(47.3 \%)$ patients in the placebo group and $18(60 \%)$ in the donepezil showing no substantial differences $(p=0.215)$ between the two groups. The baseline cortical thickness, for all brain regions considered, did not show any significant statistical differences between the two groups (Table 2).

As detailed in the Figure 1, a significant difference in the regional cortical thickness APC was observed between groups. In particular, the Placebo group compared with the Donepezil showed a higher APC in the right and left Rostral Anterior Cingulate Cortex $-1.14 \%$ vs $-0.38 \%(p=0.048)$ and $-1.28 \%$ vs $0.07 \%(p=0.032)$ respectively, in the left Caudal Anterior Cingulate Cortex $-1.07 \%$ vs $0.16 \%(p=0.033)$, in the right and left Orbitofrontal Cortex $-1.04 \%$ vs $-0.001 \%(p=0.012)$ and $-0.54 \%$ vs $0.43 \%(p$ $<0.048)$ respectively, in the right Inferior Frontal Cortex $-0.94 \%$ vs $0.23 \%(p=0.022)$, and in the right Insula $-1.06 \%$ vs $0.013 \%(p=0.010)$.

Post-hoc analysis by the mixed effects model (Figure 2) revealed that, during the 12 months of treatment period, cortical thickness was significantly decreased in the Placebo group compared with the Donepezil in the in the right Lateral Orbitofrontal cortex (difference in slope 0.0023; $p=0.026$ ), in the right Middle temporal Cortex (difference in slope $0.0027 ; p=0.027$ ) and in the right Insula (difference in slope $0.0027 ; p=0.015)$. Both the cortical thickness APC comparisons and the results on 
the mixed effect model did not survived after Bonferroni correction.

Surface differences were described in Figure 3 revealing a cortical thinning of Placebo group compared to the Donepezil in the Left Superior Temporal, Left Orbitofrontal, Right Supramarginal and Right Insula cortices. This result did not survive after FDR correction.

Power analysis

The mean (SD) cortical thickness APC for Donepezil group was -0.51 (2.18), while in the Placebo group was -0.95 (2.10). Based on these values, to have $80 \%$ power to detect a drug effect at $5 \%$ significance level $(\alpha=0.05), 187$ patients would be needed for each treatment arm. This assumes a 1 year-placebo-controlled trial with a 10\% patients drop-out rate 


\section{Discussion}

To our knowledge, this is the first study investigating the effect of donepezil treatment on regional brain cortical thickness in suspected prodromal AD patients with amnestic syndrome of hippocampal type. Our results highlight the presence of a trend of stability in the cortical thickness APC in the Anterior Cingulate Cortex, in the left Orbitofrontal Cortex, in the Inferior Frontal Cortex, and in the right Insula in suspected prodromal AD patients treated with donepezil. These findings have been furthermore supported by data obtained from the Longitudinal Mixed Model and the cortical surface comparison of cortical thickness APC between groups. Indeed, we have found a trend of reduced cortical surface in the placebo group compared with the donepezil after one year of Donepezil treatment in the following regions:

Orbitofrontal, Superior, and Caudal Middle Frontal Cortices, Superior Temporal, Supra Marginal, Precuneus, Inferior Parietal, and Insula. Some of these areas have been recognised to be of key importance since they are supposed to represent the cortical signature of Prodromal AD patients ${ }^{29,30}$. Although our results did not survive after multiple comparisons, they still remain promising since they have showed a statistical trend toward a significant impact of donepezil treatment in preserving cortical thickness in suspected prodromal AD patients. One possible reason explaining why our results did not survive to multiple comparison corrections might due to the fact that the sample power of the current study was not calculated for the present purpose but for the original aim of the Hippocampus Study Trial. Indeed, the necessary sample size to obtain a statistically significant effect in the cortical thickness APC between Placebo and Donepezil should be substantially higher, 187 patients for each arm, as revealed by the sample power analysis.

Notably, in line with a previous study revealing an increased resting state metabolism 
in the left prefrontal cortex (and in the right hippocampus) of $A D$ patients treated with Donepezil ${ }^{11}$, we also found a significant reduced cortical thinning in left Orbitofrontal Cortex and Anterior Cingulate Cortex in this group.

Previous studies investigating the donepezil effect on different structural imaging markers, such as the hippocampus, the brain lateral ventricles, and the whole brain volumes, showed contrasting results. A randomized double-blind, placebo-controlled mono-center study on 67 patients with mild-to-moderate $A D$ found a small decrease in left hippocampal volume after 24-week of donepezil compared with the placebotreated subjects ${ }^{31}$. Results from the Alzheimer's Disease Cooperative Study (ADCS) $\mathrm{MCl}$ Donepezil/Vitamin E study conducted on $131 \mathrm{MCl}$ patients showed a statistical trend towards a possible slowing of the hippocampal atrophy rate by donepezil ${ }^{12}$. A more recent study conducted by Schuff and colleagues ${ }^{32}$ on aMCI subjects reported significant differences in favour of the donepezil group for the total ventricular region and the cortical region (whole brain volumes) but not for the hippocampal volume. Recently, our group confirmed the data by Schuff and colleagues ${ }^{32}$ for ventricular and whole brain volumes. In addition, we observed a significant reduction in the annual rate of hippocampal atrophy in suspected prodromal AD patients treated with donepezil ${ }^{33}$.

Regional cortical thickness reduction is used as a predictive indicator of AD-related neurodegeneration in $\mathrm{MCl}$ subjects ${ }^{30,34}$. Our results revealed unilateral treatment differences in several brain regions that might be due to normal variation and specialization of function and structure. Brain asymmetry is believed to be evolutionally adaptive, reducing possible interference between hemispheres ${ }^{35}$.

Based on the hypothesis that donepezil may attenuate amyloid-induced neuronal 
toxicity ${ }^{36,37}$, it is conceivable that 12 months of donepezil treatment might have a neuroprotective effect on the cortex. In vitro studies demonstrated that lesions of the cholinergic nucleus basalis of Meynert promote the ex vivo synthesis of $A \beta$ precursor protein $(\mathrm{A} \beta \mathrm{PP})$ in the cerebral cortex ${ }^{38}$. Recently, an in vivo neuroimaging study described a correlation between basal forebrain atrophy and elevated cortical amyloid load in preclinical and pre-dementia stages of $A D^{39}$, thus reflecting the association found in several human autopsy studies between amyloid pathology and cholinergic atrophy in AD 40, 41, 42. Cortical amyloid accumulation might induce cholinergic cell death involving alterations in the levels of intracellular calcium and/or production of toxic and inflammatory mediators such as nitric oxide, cytokines, and reactive oxygen intermediates ${ }^{43}$. On the other hand, a cholinergic stimulation alters positively the mechanisms of amyloid processing, thus protecting neurons from neurodegeneration induced by $A \beta^{36}$.

In this study, we found a trend toward a statistical significance for cortical thinning reduction in the cortical areas innervated by the medial and lateral cholinergic pathways ${ }^{44}$ in suspected prodromal AD patients receiving one year of donepezil treatment.

One of the main strengths of the present study is the refined target population deriving from a large-scale community-based multi-centre cohort of subjects included on the basis of the FCSRT, a memory test reported to be highly correlated with hippocampal volume and CSF levels changes of the Alzheimer type ${ }^{18,19}$. The study presents potential limitations. First of all, the data used in the present research were not specifically powered for the aims of the present study, thus reducing the significance of the results. The protocol of the study did not include information on the settings of the subjects of the population or on race and ethnicity 
characteristics or data in terms of lifestyle. The latter, in particular, given its practical aspects - nutrition, hydration, and physical activity - has become significant in terms of $A D$ prevention. We did not collect sufficient data on $A P O E \varepsilon 4$ genotype to exclude the hypothesis that our results were not significantly impacted by the presence of $A P O E \& 4$ genotype. Finally, the subjects have not been followed for a long enough period of time in order to determine the incidence of incipient AD in each group. Overall, our findings support the hypothesis that cortical thickness might be used as surrogate outcome in pre-dementia AD clinical trials. Moreover, our findings suggest that donepezil may have an impact on cortical morphology in prodromal AD patients. A potentially disease-modifying effect of approved cholinesterase inhibitors represents a result of pivotal clinical interest. Further studies are needed to confirmed this first investigation. Moreover, established measurement of the basal forebrain cholinergic nuclei volume, such as the nucleus basalis of Meynert and its subnuclei, as well as their in vivo white matter connections to subcortical and cortical areas, are of interest to detect the specific donepezil effect on the brain's cholinergic system. 


\section{Acknowledgement}

We would like to acknowledge Prof Touchon and Dr Aimeur who participated in the study. We would like to acknowledge Dr Line Garnero who deceased during the study, without her support and experience this study would never have been undertaken.

Funding and Disclosure: The clinical trial with donepezil leading to the analysed dataset was supported by Eisai SAS France. Eisai SAS France supported for data collection and for the investigation of the primary endpoint of the study.

Dr Cavedo has received funding from the grant Conv. n.130/GR-2011-02350494 funded by the Italian Ministry of Health (Bando Giovani Ricercatori 2011-2012) ; Dr Dubois has received consulting fees from Eli Lilly, Dr Dubois have received Grant support from Pfizer in 2012 ; Dr Colliot declares no competing financial interests related to the present article, he has received consulting fees from Guerbet and lecture fees from Lundbeck and Roche. Dr Lista has received lecture fees from Roche. Dr Girard is consulting for OLEA MEDICAL, he has received travel funding for scientific congress (below 10000 euros per year). Dr Hampel and Dr Lista are supported by the AXA Research Fund, the Universite Pierre et Marie Curie and the "Association pour la Recherche sur Alzheimer", Paris, France. Dr Hampel's research leading to these results has received funding from the program "Investissements d'avenir" ANR-10-IAIHU-06. Dr Hampel declares no competing financial interests related to the present article. During the last 36 months Dr Hampel has received lecture honoraria and/or research grants (paid to the institution) and/or travel funding and/or participated in scientific advisory boards and/or as a consultant to diagnostic, biotechnology and pharmaceutical companies involved in the manufacture and marketing of biomarkers and/or diagnostics and/or drugs or medicinal products for 
cognitive impairment and Alzheimer's disease including Boehringer-Ingelheim, Bristol-Myers Squibb, Elan Corporation, Novartis, Eisai Inc., Pfizer, Sanofi-Aventis, Roche Pharmaceuticals and Diagnostics, GE Healthcare, Avid, Eli Lilly and Company, GlaxoSmithKline-Biologicals, Jung-Diagnostics, Cytox, Anavex, Axovant, Biogen Idec, Oryzon Genomics. He is co-inventor in patents relating to biological markers and/or diagnostics and has not received any royalties Dr Croisile, Tisserand, Bonafe, Ousset, Rouaud, Ricolfi, Vighetto, Pasquier, Galluzzi, Delmaire, Ceccaldi, Lehericy, Duveau, Chupin, Sarazin, Dormont declare no competing financial interests.

\section{Hippocampus study group:}

O. GODEFROY (Amiens), H. DERAMOND (Amiens), F. ETCHARRY BOUYX (Angers), A. PASCO PAPON (Angers), JF. DARTIGUES (Bordeaux), M. ALLARD (Bordeaux), O. ROUAUD (Dijon), F. RICOLFI (Dijon), O. MOREAUD (Grenoble), A. KRAINIK (Grenoble), F. PASQUIER (Lille), S. BOMBOIS (Lille), C. DELMAIRE (Lille), P. COURATIER (Limoges), A. MAUBON (Limoges), A. VIGHETTO (Lyon), B. CROISILE (Lyon), G. LOUIS-TISSERAND (Lyon), M. CECCALDI (Marseille), N. GIRARD (Marseille), B. MICHEL (Marseille), MP. PERETTI (Marseille), J. TOUCHON (Montpellier), A. BONAFE (Montpellier), A. BENETOS (Nancy), G BARROCHE (Nancy), S. BRACARD (Nancy), M. VERCELLETTO (Nantes), E. CALVIER AUFFRAY ( Nantes), P. ROBERT (Nice), S. CHANALET (Nice), B. DUBOIS and H. HAMPEL (Paris), D. DORMONT (Paris), S. LEHERICY (Paris), J. HUGON (Paris), M.P. PANCRAZI (Paris), D. REIZINE (Paris), R. GIL (Poitiers), P. VANDERMARCQ (Poitiers), J.L. NOVELLA (Reims), L. PIEROT (Reims), S. BELLIARD (Rennes), M. CARSIN (Rennes), O. MARTINAUD (Rouen), E. GERARDIN (Rouen), B. LAURENT 
(Saint Etienne), FG. BARRAL (Saint Etienne), F. SELLAL (Strasbourg), PJ. OUSSET

(Toulouse), M. PUEL (Toulouse), A. AIT AMEUR (Toulouse), H. DUMAS (Toulouse),

K. MONDON (Tours), JP. COTTIER (Tours).

Clinical Points

- Cortical thickness measurements may represent reliable surrogate markers of disease progression and underlying Alzheimer's Disease at prodromal stages

- Our data indicate that Donepezil treatment may reduce cortical thickness annualized percent changes mainly in cortical areas associated to the cholinergic system supporting the hypothesis that Donepezil may impact on brain structure/morphology 


\section{References}

1. Johannsen $\mathrm{P}$, Salmon $\mathrm{E}$, Hampel $\mathrm{H}$, et al. Assessing therapeutic efficacy in a progressive disease: a study of donepezil in Alzheimer's disease. CNS Drugs $2006 ; 20(4): 311-325$.

2. Crane PK, Doody RS. Donepezil treatment of patients with $\mathrm{MCl}$ : a 48-week randomized, placebo- controlled trial. Neurology 2009 Nov;73(18):1514-1515; author reply 1515-1516.

3. Doody RS, Ferris SH, Salloway S, et al. Donepezil treatment of patients with MCl: a 48-week randomized, placebo-controlled trial. Neurology 2009 May;72(18):1555-1561.

4. Doody RS, Ferris S, Salloway S, et al. Safety and tolerability of donepezil in mild cognitive impairment: open-label extension study. Am J Alzheimers Dis Other Demen 2010 Mar;25(2):155-159.

5. Feldman H, Gauthier S, Hecker J, et al. A 24-week, randomized, double-blind study of donepezil in moderate to severe Alzheimer's disease. Neurology 2001 Aug;57(4):613-620.

6. Feldman H, Gauthier S, Hecker J, et al. Efficacy of donepezil on maintenance of activities of daily living in patients with moderate to severe Alzheimer's disease and the effect on caregiver burden. J Am Geriatr Soc 2003 Jun;51(6):737-744.

7. Feldman H, Gauthier S, Hecker J, et al. Efficacy and safety of donepezil in patients with more severe Alzheimer's disease: a subgroup analysis from a randomized, placebo-controlled trial. Int J Geriatr Psychiatry 2005 Jun;20(6):559-569. 
8. Gauthier S, Feldman H, Hecker J, et al. Efficacy of donepezil on behavioral symptoms in patients with moderate to severe Alzheimer's disease. Int Psychogeriatr 2002 Dec;14(4):389-404.

9. Petersen RC, Thomas RG, Grundman M, et al. Vitamin E and donepezil for the treatment of mild cognitive impairment. N Engl J Med 2005 Jun;352(23):2379-2388.

10. Salloway S, Ferris S, Kluger A, et al. Efficacy of donepezil in mild cognitive impairment: a randomized placebo-controlled trial. Neurology 2004 Aug;63(4):651-657.

11. Teipel SJ, Drzezga A, Bartenstein P, Möller HJ, Schwaiger M, Hampel H. Effects of donepezil on cortical metabolic response to activation during (18)FDG-PET in Alzheimer's disease: a double-blind cross-over trial. Psychopharmacology (Berl) 2006 Jul;187(1):86-94.

12. Jack CR, Jr., Petersen RC, Grundman M, et al. Longitudinal MRI findings from the vitamin E and donepezil treatment study for MCI. Neurobiol Aging 2008 September;29(9):1285-1295.

13. Schuff N, Suhy J, Goldman R, et al. An MRI substudy of a donepezil clinical trial in mild cognitive impairment. Neurobiol Aging 2011 Dec;32(12):2318 e2331-2341.

14. Dubois B, Chupin M, Hampel $\mathrm{H}$, et al. Donepezil decreases annual rate of hippocampal atrophy in suspected prodromal Alzheimer's disease. Alzheimers Dement 2015 Jan 14.

15. Teipel SJ, Grothe M, Lista S, Toschi N, Garaci FG, Hampel H. Relevance of magnetic resonance imaging for early detection and diagnosis of Alzheimer disease. Med Clin North Am 2013 May;97(3):399-424. 
16. Hampel H, Bürger K, Teipel SJ, Bokde AL, Zetterberg H, Blennow K. Core candidate neurochemical and imaging biomarkers of Alzheimer's disease. Alzheimers Dement 2008 Jan;4(1):38-48.

17. Teipel SJ, Meindl T, Grinberg L, Heinsen H, Hampel H. Novel MRI techniques in the assessment of dementia. Eur J Nucl Med Mol Imaging 2008 Mar;35 Suppl 1:S58-69.

18. Wagner M, Wolf S, Reischies FM, et al. Biomarker validation of a cued recall memory deficit in prodromal Alzheimer disease. Neurology 2012 Feb;78(6):379-386.

19. Sarazin M, Chauviré V, Gerardin E, et al. The amnestic syndrome of hippocampal type in Alzheimer's disease: an MRI study. J Alzheimers Dis 2010;22(1):285-294.

20. Rosen WG, Mohs RC, Davis KL. A new rating scale for Alzheimer's disease. Am J Psychiatry 1984 Nov;141(11):1356-1364.

21. Dean C. Delis JHK, Edith Kaplan, Beth Anne Ober Thompkins. CVLT, California Verbal Learning Test:Adult Version : Manual.

22. Reitan MW, D. The Halstead-Reitan Neuropsychological Test Battery: Theory and clinical interpretation; 1993.

23. Benton AL. The revised visual retention test: The Psychological Corporation, New York; 1974.

24. Bernal-Rusiel JL, Greve DN, Reuter M, Fischl B, Sabuncu MR, Initiative AsDN. Statistical analysis of longitudinal neuroimage data with Linear Mixed Effects models. Neuroimage 2013 Feb;66:249-260.

25. Reuter M, Rosas HD, Fischl B. Highly accurate inverse consistent registration: a robust approach. Neuroimage 2010 Dec;53(4):1181-1196. 
26. Reuter M, Schmansky NJ, Rosas HD, Fischl B. Within-subject template estimation for unbiased longitudinal image analysis. Neuroimage 2012 Jul;61(4):1402-1418.

27. Desikan RS, Ségonne F, Fischl B, et al. An automated labeling system for subdividing the human cerebral cortex on MRI scans into gyral based regions of interest. Neuroimage $2006 \mathrm{Jul} ; 31(3): 968-980$.

28. G.F.Templeton. A Two-Step Approach for Transforming Continuous Variables to Normal: Implications and Recommendations for IS Research. Communications of the AIS 2011;28.

29. Bakkour A, Morris JC, Dickerson BC. The cortical signature of prodromal AD: regional thinning predicts mild AD dementia. Neurology 2009 Mar 24;72(12):1048-1055.

30. Dickerson BC, Wolk DA, Initiative AsDN. MRI cortical thickness biomarker predicts AD-like CSF and cognitive decline in normal adults. Neurology 2012 Jan;78(2):84-90.

31. Krishnan KR, Charles HC, Doraiswamy PM, et al. Randomized, placebocontrolled trial of the effects of donepezil on neuronal markers and hippocampal volumes in Alzheimer's disease. Am J Psychiatry 2003 Nov;160(11):2003-2011.

32. Schuff N, Woerner N, Boreta L, et al. MRI of hippocampal volume loss in early Alzheimer's disease in relation to ApoE genotype and biomarkers. Brain : a journal of neurology 2009;132(Pt 4):1067-1077.

33. Dubois B, Chupin M, Hampel H, et al. Donepezil decreases annual rate of hippocampal atrophy in suspected prodromal Alzheimer's disease. Alzheimers Dement 2015 Jan. 
34. Lerch JP, Pruessner J, Zijdenbos AP, et al. Automated cortical thickness measurements from MRI can accurately separate Alzheimer's patients from normal elderly controls. Neurobiol Aging 2008 Jan;29(1):23-30.

35. Toga AW, Thompson PM. Mapping brain asymmetry. Nat Rev Neurosci 2003 Jan;4(1):37-48.

36. Svensson AL, Nordberg A. Tacrine and donepezil attenuate the neurotoxic effect of A beta(25-35) in rat PC12 cells. Neuroreport 1998 May;9(7):15191522.

37. Wolf BA, Wertkin AM, Jolly YC, et al. Muscarinic regulation of Alzheimer's disease amyloid precursor protein secretion and amyloid beta-protein production in human neuronal NT2N cells. J Biol Chem 1995 Mar;270(9):4916-4922.

38. Wallace W, Ahlers ST, Gotlib J, et al. Amyloid precursor protein in the cerebral cortex is rapidly and persistently induced by loss of subcortical innervation. Proc Natl Acad Sci U S A 1993 Sep;90(18):8712-8716.

39. Grothe MJ, Ewers M, Krause B, Heinsen H, Teipel SJ, Initiative AsDN. Basal forebrain atrophy and cortical amyloid deposition in nondemented elderly subjects. Alzheimers Dement 2014 Jan.

40. Perry EK, Tomlinson BE, Blessed G, Bergmann K, Gibson PH, Perry RH. Correlation of cholinergic abnormalities with senile plaques and mental test scores in senile dementia. Br Med J 1978 Nov;2(6150):1457-1459.

41. Beach TG, McGeer EG. Senile plaques, amyloid beta-protein, and acetylcholinesterase fibres: laminar distributions in Alzheimer's disease striate cortex. Acta Neuropathol 1992;83(3):292-299. 
42. Arendt T, Bigl V, Tennstedt A, Arendt A. Neuronal loss in different parts of the nucleus basalis is related to neuritic plaque formation in cortical target areas in Alzheimer's disease. Neuroscience 1985 Jan;14(1):1-14.

43. Kar S, Slowikowski SP, Westaway D, Mount HT. Interactions between betaamyloid and central cholinergic neurons: implications for Alzheimer's disease. J Psychiatry Neurosci 2004 Nov;29(6):427-441.

44. Selden NR, Gitelman DR, Salamon-Murayama N, Parrish TB, Mesulam MM. Trajectories of cholinergic pathways within the cerebral hemispheres of the human brain. Brain : a journal of neurology 1998;121 ( Pt 12)(Pt 12):22492257. 


\section{Figures}

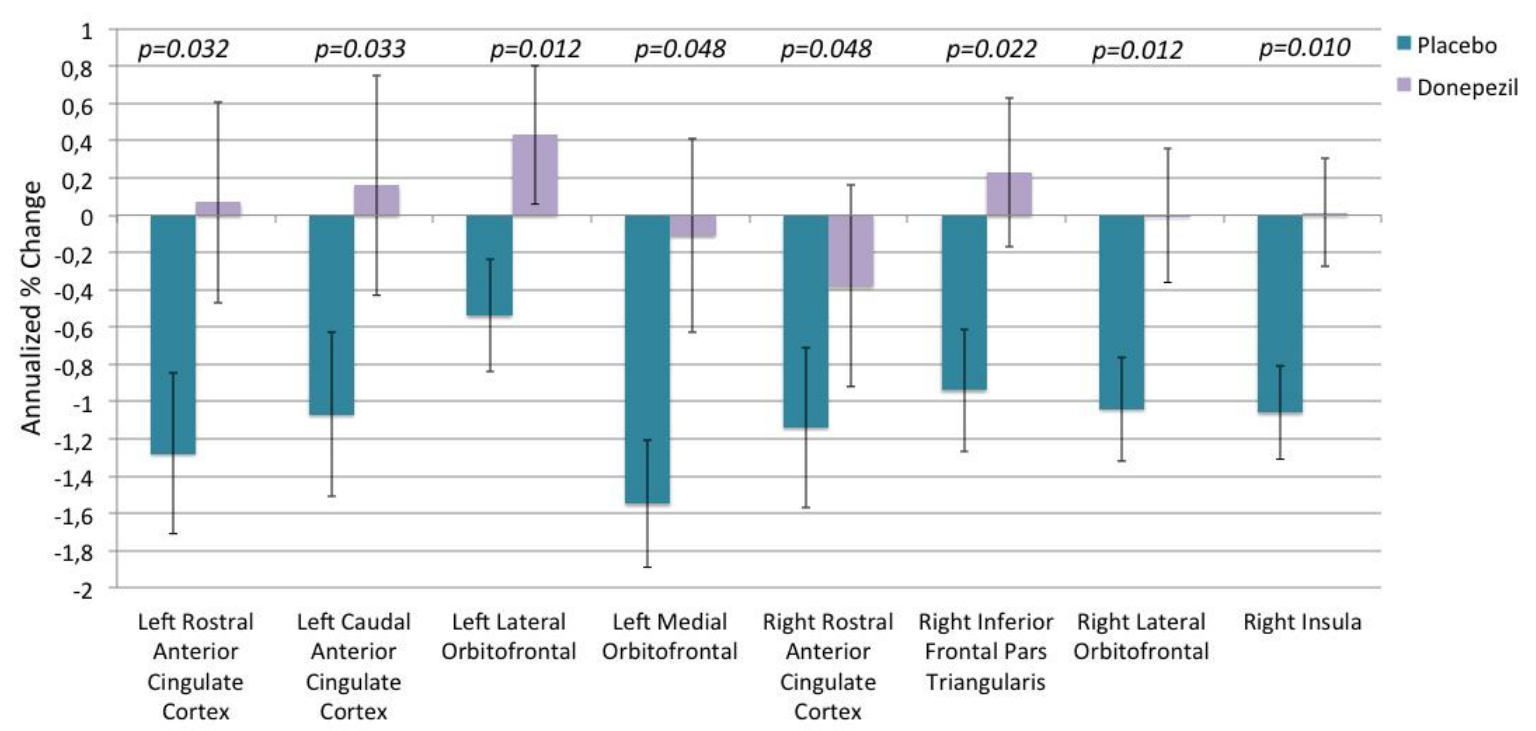

Figure 1. Significant differences in the Left and Right Brain Cortical Thickness Annualized Percentage Changes. Patients treated with Donepezil showed an increase or stable change of the frontal and insula cortical thicknesses compared to the Placebo group revealing a cortical thinning in all the cortical regions. Numbers indicate means (errors standard) of Annual Percentage Change; $p$ values denote the significance at Wilcoxon-Mann-Whitney test. 

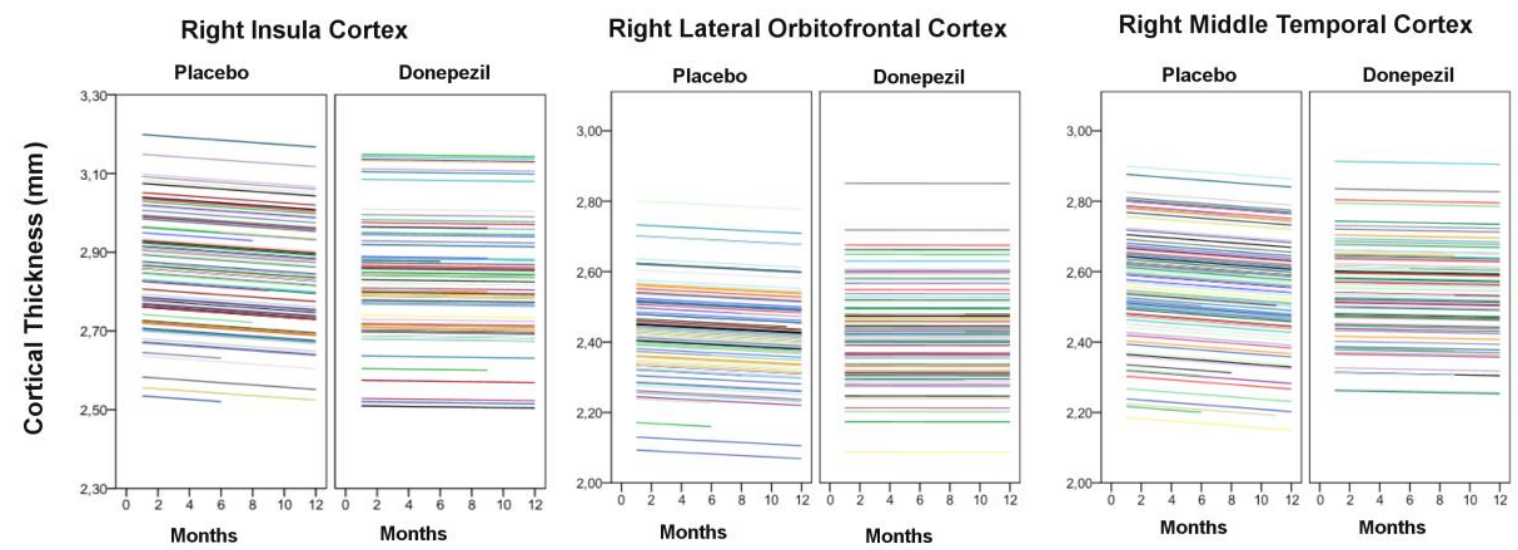

Figure 2. Individual trajectories of Cortical Thickness change. Graphs display significant changes over time resulted from the Linear Mixed Effects regression Models with random intercepts in the donepezil and placebo groups based on cortical thickness measurements from baseline (Month 0 ) to follow-up scan. 


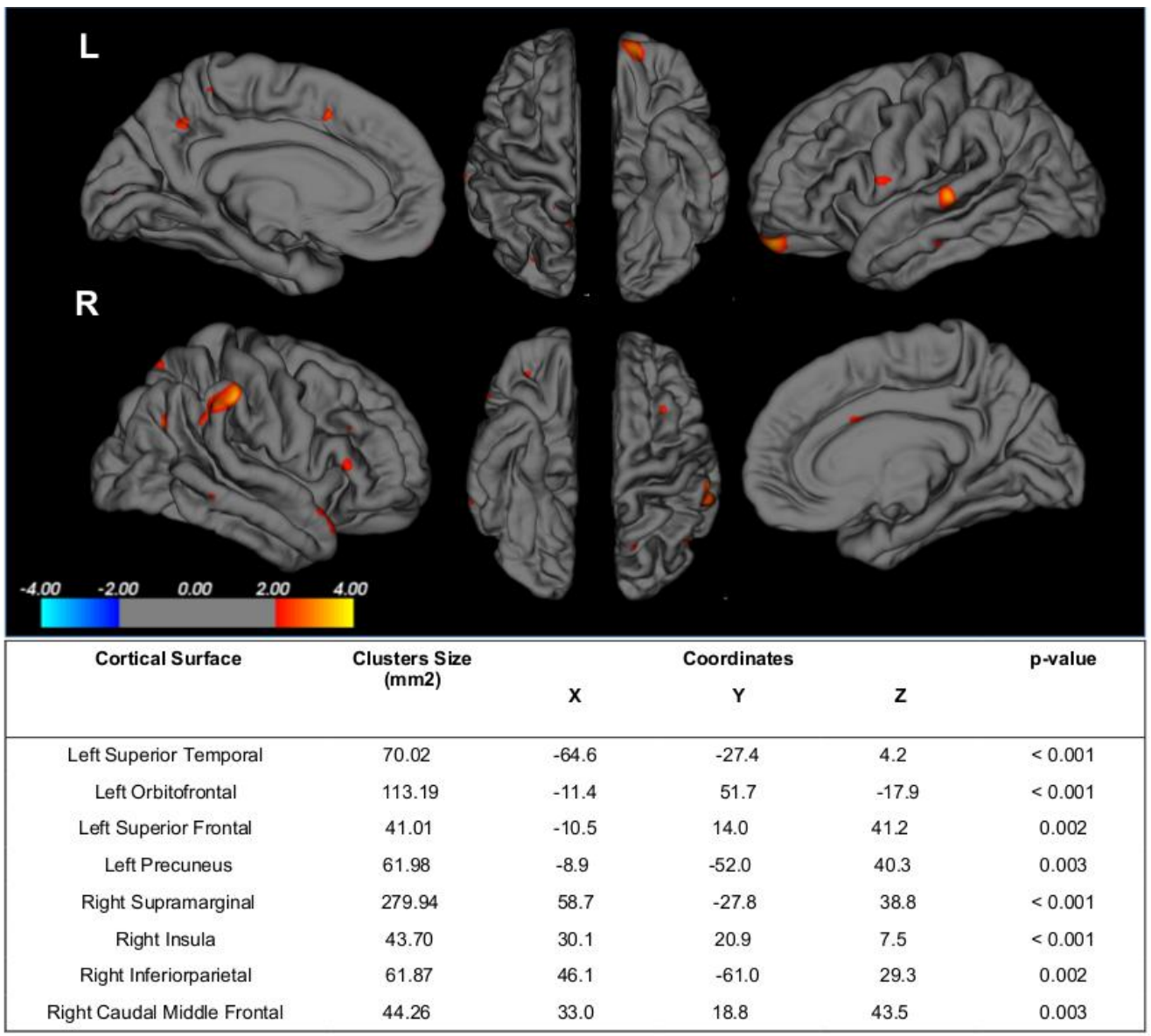

Figure 3. Surface differences between placebo and donepezil groups detected by the Linear Mixed Effects Model, results are FDR uncorrected. 


\section{Tables}

Table 1: Baseline Demographic and Clinical Characteristics of patients performing baseline and follow-up MRI. Means and standard deviations are reported for continuous variables, numbers and percentages for the dichotomous ones. P-values denote significant differences at ANOVA and Chi-square tests.

\begin{tabular}{|c|c|c|c|}
\hline & $\begin{array}{l}\text { Placebo } \\
(n=92)\end{array}$ & $\begin{array}{l}\text { Donepezil } \\
(\mathrm{n}=81)\end{array}$ & P Value \\
\hline Age, years & $73.17(6.63)$ & $73.24(6.67)$ & 0.966 \\
\hline \multicolumn{4}{|l|}{ Sex, n (\%) } \\
\hline Male & $44(47.8)$ & $39(48.1)$ & 0.544 \\
\hline Female & $48(52.1)$ & $42(51.8)$ & \\
\hline APOE $\varepsilon 4$ carriers, $n(\%)$ & $18(47.3)$ & $18(60)$ & 0.215 \\
\hline Missing & 54 & 43 & \\
\hline \multicolumn{4}{|l|}{ Education, $\mathrm{n}(\%)$} \\
\hline No education & $1(0.01)$ & $0(0.0)$ & 0.330 \\
\hline Primary & $7(7.6)$ & $8(9.8)$ & \\
\hline Certificate of Primary Education & $43(46.7)$ & $30(37)$ & \\
\hline Secondary & $14(15.2)$ & $21(25.9)$ & \\
\hline Higher education & 27 (29.3) & $22(27.1)$ & \\
\hline Follow-up MRI (months) & $9.65(4.78)$ & $10.07(4.43)$ & 0.537 \\
\hline FCSRT (Free recall) & $11.32(5.61)$ & $12.21(5.40)$ & 0.289 \\
\hline FCSRT (Total recall) & $29.55(9.93)$ & $31.37(8.91)$ & 0.210 \\
\hline $\begin{array}{l}\text { Hamilton Rating Scale for } \\
\text { Depression }\end{array}$ & $3.22(2.94)$ & $2.83(2.49)$ & 0.352 \\
\hline
\end{tabular}




\begin{tabular}{|l|l|l|l|}
\hline ADAS-COG-MCI & $12.32(4.40)$ & $12.32(4.61)$ & 0.997 \\
\hline MMSE & & & \\
\hline 3 Tesla MRI (\%) & $25.86(2.79)$ & $25.94(2.26)$ & 0.838 \\
\hline
\end{tabular}

Abbreviations: $\mathrm{APOE}=$ Apolipoprotein $\mathrm{E}, \mathrm{FCSRT}=$ Free and Cued Selective Reminding Test, MRI= Magnetic Resonance Imaging. 
Table 2: Baseline Right and Left Cortical thickness (mm) of 173 patients.

Means and standard deviations are reported, $\mathrm{P}$ Values denote significant differences at ANOVA.

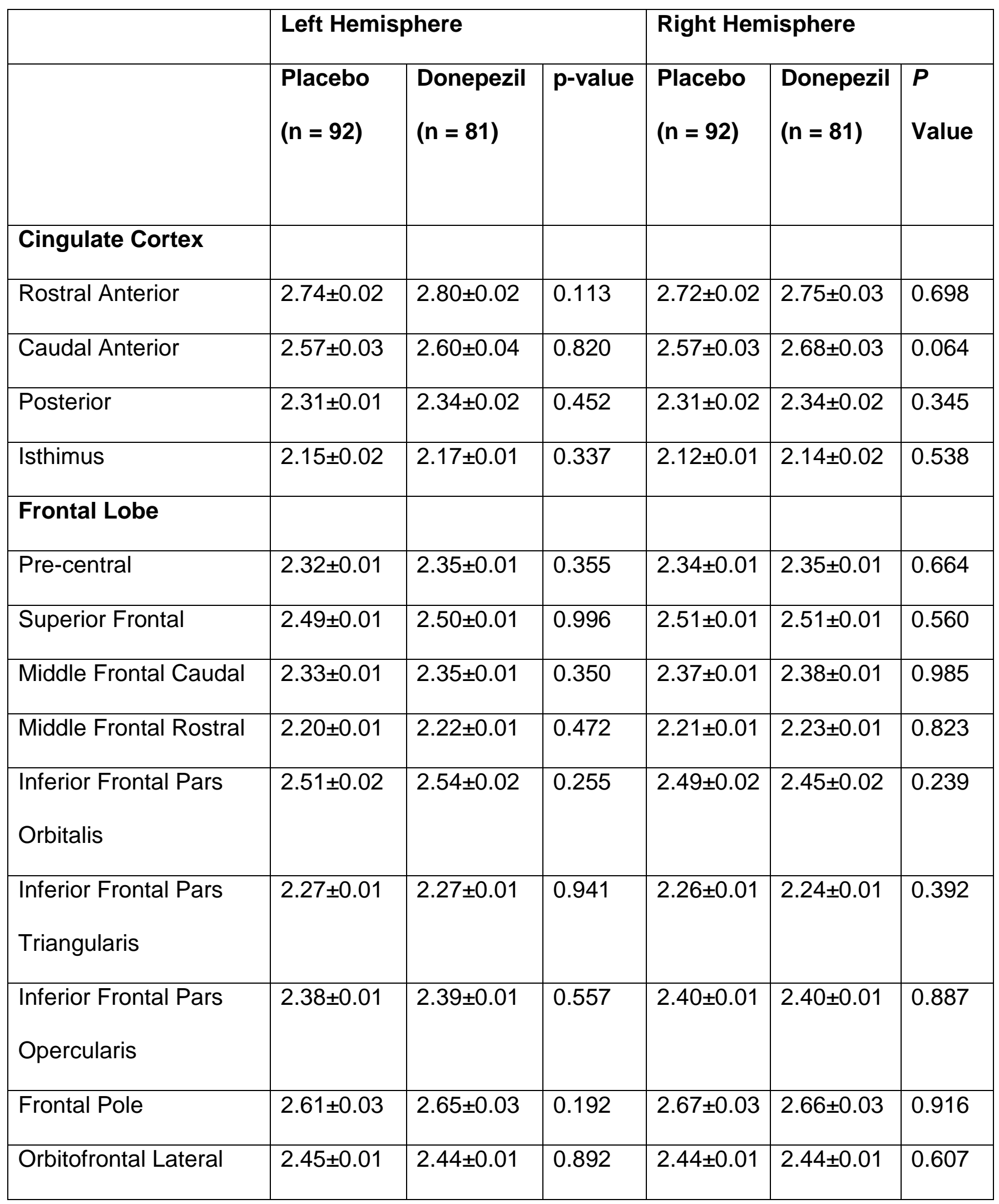




\begin{tabular}{|l|l|l|l|l|l|l|}
\hline Orbitofrontal Medial & $2.36 \pm 0.02$ & $2.36 \pm 0.01$ & 0.308 & $2.34 \pm 0.01$ & $2.36 \pm 0.01$ & 0.143 \\
\hline Parietal & & & & & & \\
\hline Post-Central & $1.95 \pm 0.01$ & $1.99 \pm 0.02$ & 0.460 & $1.98 \pm 0.01$ & $2.00 \pm 0.01$ & 0.472 \\
\hline Supra Marginal & $2.31 \pm 0.01$ & $2.32 \pm 0.01$ & 0.885 & $2.33 \pm 0.01$ & $2.34 \pm 0.01$ & 0.848 \\
\hline Superior Parietal & $2.01 \pm 0.01$ & $2.04 \pm 0.01$ & 0.182 & $2.05 \pm 0.01$ & $2.06 \pm 0.01$ & 0.814 \\
\hline Inferior Parietal & $2.22 \pm 0.01$ & $2.26 \pm 0.01$ & 0.166 & $2.22 \pm 0.01$ & $2.25 \pm 0.01$ & 0.164 \\
\hline Precuneus & $2.12 \pm 0.01$ & $2.14 \pm 0.01$ & 0.541 & $2.12 \pm 0.01$ & $2.13 \pm 0.01$ & 0.798 \\
\hline Temporal & & & & & & \\
\hline Inferior Temporal & $2.56 \pm 0.01$ & $2.58 \pm 0.02$ & 0.447 & $2.57 \pm 0.01$ & $2.57 \pm 0.01$ & 0.986 \\
\hline Superior Temporal & $2.5 \pm 0.1$ & $2.5 \pm 0.1$ & 0.980 & $2.5 \pm 0.1$ & $2.5 \pm 0.1$ & 0.852 \\
\hline Transverse Temporal & $2.25 \pm 0.02$ & $2.22 \pm 0.02$ & 0.770 & $2.26 \pm 0.02$ & $2.27 \pm 0.02$ & 0.824 \\
\hline Middle Temporal & $2.59 \pm 0.01$ & $2.60 \pm 0.01$ & 0.778 & $2.56 \pm 0.01$ & $2.55 \pm 0.01$ & 0.504 \\
\hline Temporal Pole & $3.3 \pm 0.3$ & $3.2 \pm 0.3$ & 0.376 & $3.32 \pm 0.04$ & $3.31 \pm 0.03$ & 0.991 \\
\hline Entorhinal & $2.94 \pm 0.04$ & $2.88 \pm 0.04$ & 0.554 & $2.84 \pm 0.05$ & $2.87 \pm 0.04$ & 0.669 \\
\hline Para-hippocampal & $2.38 \pm 0.03$ & $2.41 \pm 0.03$ & 0.936 & $2.34 \pm 0.02$ & $2.28 \pm 0.04$ & 0.498 \\
\hline Fusiform & $2.43 \pm 0.01$ & $2.45 \pm 0.01$ & 0.322 & $2.41 \pm 0.01$ & $2.43 \pm 0.01$ & 0.270 \\
\hline Occipital & $1.75 \pm 0.01$ & $1.78 \pm 0.01$ & 0.250 & $1.74 \pm 0.01$ & $1.75 \pm 0.01$ & 0.725 \\
\hline Lateral Occipital & $2.03 \pm 0.01$ & $2.05 \pm 0.01$ & 0.713 & $2.02 \pm 0.01$ & $2.03 \pm 0.01$ & 0.887 \\
\hline Cuneus & $1.56 \pm 0.01$ & $1.56 \pm 0.01$ & 0.940 & $1.51 \pm 0.01$ & $1.51 \pm 0.01$ & 0.702 \\
\hline Peri-Calcarine & $1.85 \pm 0.01$ & $1.86 \pm 0.01$ & 0.718 & $1.83 \pm 0.01$ & $1.82 \pm 0.01$ & 0.889 \\
\hline Lingual & $2.81 \pm 0.01$ & $2.80 \pm 0.01$ & 0.748 & $2.86 \pm 0.01$ & $2.82 \pm 0.02$ & 0.111 \\
\hline Insula & & & & & & \\
\hline & & & & & & \\
\hline
\end{tabular}

